\title{
Constrained Interpolation with Implicit Plane Cubic A-Splines
}

\author{
S. Behar-Jequín, J. Estrada-Sarlabous, and V. Hernández-Mederos \\ ${ }^{1}$ Facultad de Matemática y Computación, Universidad de la Habana, Cuba \\ ${ }^{2}$ Instituto de Cibernética, Matemática y Física, CITMA, Cuba
}

\begin{abstract}
In this work we focus on the following problem: given an ordered set of points in the plane lying on one side of a given boundary polygon, construct a smooth curve interpolating these points, such that the curve lies on the same side of the polygon as the data.

We have developed a $G^{2}$-continuous cubic A-spline scheme smoothing the polygon defined by the line segments joining consecutive data points, such that the spline curve lies completely on the same side of the boundary polygon as the data. The proposed A-spline scheme provides an efficient method for generating a smooth robot's path that avoids corners or polygonal objects for a given planned path, for designing a smooth curve on a polygonal piece of material, etc.
\end{abstract}

Keywords: Constrained interpolation; $G^{2}$-continuous cubic A-spline; Shape preserving spline.

Mathematics Subject Classification: 65D07 (splines), 65D05 (interpolation), 65D17 (Computer Aided Design).

\section{Introduction}

Polynomial and rational parametric spline curves are the most popular tools to approximate a given (finite) set of points in the plane. More recently, a new approach is been intensively researched: the approximating spline curves are defined as piecewise plane algebraic curves, represented by their implicit (polynomial) equation. These splines are called A-splines.

The A-spline curves include as particular cases the polynomial and the rational parametric spline curves. Since they are more general curves, for a fixed degree of the spline we have more free parameters to impose additional interpolation conditions or geometric constraints (see [5, 6], 1, 2]).

In this work we consider the following problem: given an ordered set of points $\mathcal{D}$ in the plane lying on one side of a previously given polygon $\mathcal{B}$, construct a smooth curve $\mathcal{A}$ interpolating the points of $\mathcal{D}$, such that $\mathcal{A}$ lies on the same side of $\mathcal{B}$ as the data from $\mathcal{D}$. A typical situation, where this kind of constrained interpolation arises, is the design of a smooth robot's path avoiding corners and/or polygonal obstacles.

The proposed A-spline curve scheme happens to be very suitable to solve the above mentioned constrained interpolation problem, since this problem may be 
translated to the interpolation and/or approximation of the data from $\mathcal{D}$ using a smooth A-spline curve with error bounds depending on its distance to $\mathcal{B}$.

\section{Algorithm}

For the sake of a better understanding, we give here a brief description of the algorithm:

Input: An ordered set of points in the plane such that the polygon $\mathcal{B}$ defined by joining two consecutive points represents the boundary. An ordered set of points in the plane $\mathcal{D}$, such that the polygon $\mathcal{C}$ defined by joining two consecutive points represents a linear approximation of the path to be designed. $\mathcal{C}$ may touch $\mathcal{B}$, but it does not cross $\mathcal{B}$ transversally.

Output: A smooth ( $G^{2}$-continuous) cubic A-spline curve $\mathcal{A}$ interpolating the vertex of $\mathcal{C}$, such that $\mathcal{A}$ lies in the same side as the points of $\mathcal{D}$ with respect to polygon $\mathcal{B}$.

\section{Algorithm}

\section{Step 1. For each side $l_{i}$ of $\mathcal{C}$ do:}

- Assign tangent vectors and curvature values to the endpoints of $l_{i}$ as proposed in [3] and [4].

- If $l_{i}$ is non convex, insert in the list of vertices of $\mathcal{C}$ the midpoint of $l_{i}$, as a new vertex. Assign a tangent vector to this new vertex (inflection point) and set equal to 0 the corresponding curvature value (see subsection 3.2). Delete $l_{i}$ from the list of sides of $\mathcal{C}$ and insert in this list the two consecutive sides obtained by subdividing $l_{i}$ at its midpoint.

\section{Step 2. For each vertex $v_{j}$ of $\mathcal{C}$ do:}

- If the distance from $v_{j}$ to $\mathcal{B}$ is smaller than some previously fixed threshold, then correct the tangent vector and curvature value assigned to $v_{j}$ as we propose in subsection 3.1 .

\section{Step 3. For each side $l_{i}$ of $\mathcal{C}$ do:}

- Construct the triangle $T_{i}$ (see section 4.1), using the tangent vectors assigned to the endpoints of $l_{i}$.

- Compute the parameters $\kappa_{j}^{i}, j=0,2$ fulfilling the interpolation of the curvature values assigned to the endpoints of $l_{i}$ (see subsections 3.1 and 4.2).

- Compute the Hausdorff distance from $l_{i}$ to $\mathcal{B}, d_{i}$.

- Compute, using $d_{i}$ and $T_{i}$, a parameter value $\delta_{i}$ such that the corresponding curve section $\mathcal{A}_{i}$ of the A-spline $\mathcal{A}$ does not cross $\mathcal{B}$ (see subsection 4.5).

- Insert $\left(T_{i}, \delta_{i}, \kappa_{j}^{i}\right)$ in the list of parameters $\mathcal{P}$ that characterize sections of $\mathcal{A}$.

Step 4. For each side $l_{i}$ of $\mathcal{C}$ do:

- Render section $\mathcal{A}_{i}$ using the parameters contained in $\mathcal{P}$. 


\section{Some Notations and Preliminaries}

In this paper we will assume:

- The line segments of $\mathcal{C}$ lie on the same side of polygon $\mathcal{B}$ as the data.

- If a vertex of $\mathcal{B}$ lies on $\mathcal{C}$ then, it is also a vertex of $\mathcal{C}$.

- If two consecutive vertices of $\mathcal{C}$ lie on $\mathcal{B}$, they belong to the interior of the same line segment $l$ of $\mathcal{B}$.

\subsection{Derivative Data}

The computation of slope and curvature values presented in the $G^{2}$ piecewise rational cubic shape preserving scheme in [3] has several nice properties. It gives a spline curve with the minimum number of inflection points consistent with the data and it reproduces circular arcs, when the data come from a circle. Therefore, we assign initially to each vertex $Q_{i}$ of the polygon $\mathcal{C}$ a slope $t^{i}$ of the tangent line and a curvature value $k^{i}$ of $\mathcal{A}$ at $Q_{i}$ as proposed in [3].

Since the method in 3 . was not developed to include constraints, for those vertices of $\mathcal{C}$ that are close to the boundary $\mathcal{B}$ it is necessary to make some adaptations, see for instance [4].

In this subsection we propose some specific modifications to what is given in 3 and 4 . Our generalization fits well in the A-spline scheme and reflects better the nature of the problem to be solved.

For the vertices of $\mathcal{C}$ which are on the boundary $\mathcal{B}$, we correct these values as follows:

- If $Q_{i}$ is a vertex on $\mathcal{B}$, but $Q_{i-1}$ and $Q_{i+1}$ are not on the boundary $\mathcal{B}$, the curvature value of $\mathcal{A}$ at $Q_{i}$ remains as in [3], but the assigned slope will be changed according to two cases:

- If $Q_{i}$ coincides with a vertex of $\mathcal{B}$, we set the slope of $\mathcal{A}$ at $Q_{i}$ equal to the slope of the line perpendicular to the bisector to the angle determined by the line segments of $\mathcal{B}$ incident at $Q_{i}$ (see $Q_{i}=P$ in Fig. 1).

- If $Q_{i}$ is in the interior of a line segment $l$ of $\mathcal{B}$, we set the slope of $\mathcal{A}$ at $Q_{i}$ equal to the slope of $l$ (see $Q_{i}=S$ in Fig. 1).

- If $Q_{i}, Q_{i+1}$ are two consecutive vertices of $\mathcal{C}$ lying on the interior of the same line segment $l$ of $\mathcal{B}$, we set the slope of $\mathcal{A}$ at $Q_{i}$ and at $Q_{i+1}$ equal to the slope of $l$, and the curvature values $\mathcal{A}$ are set equal to 0 (see $Q_{i}=Q, Q_{i+1}=R$ in Fig. 11).

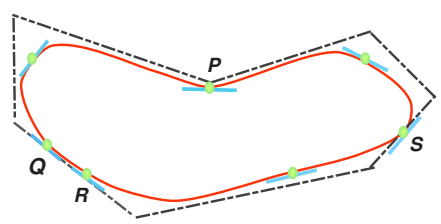

Fig. 1. Assigning tangent directions to the vertices of $\mathcal{C}$ 
Finally, if the distance from $Q_{i}$ to $\mathcal{B}$ is smaller than some previously fixed threshold, we propose to assign as slope of the tangent line of $\mathcal{A}$ at $Q_{i}$ a convex linear combination of the slope computed after the method in [3] with the slope proposed above, applied to the projection of $Q_{i}$ on $\mathcal{B}$. The weight in the convex linear combination of the slope arising from [3] is proportional to the distance of $Q_{i}$ to $\mathcal{B}$. The curvature value of $\mathcal{A}$ at $Q_{i}$ may remain as in 3 .

\subsection{Convexity of an Edge}

Definition 1. Given two consecutive vertices of $\mathcal{C}$, we call the edge passing through them convex if the associated tangent vectors point to opposite sides of the edge. Otherwise, we call the edge non convex.

In the non convex case, the midpoint of the vertices $Q_{i}$ and $Q_{i+1}$ is inserted in $\mathcal{C}$ (for the position of the inflection point of $\mathcal{A}$ ) and as slope of the tangent line of $\mathcal{A}$ at this new vertex, we assign the slope of the line perpendicular to the bisector of the angle determined by the tangent vectors associated to $Q_{i}$ and to $Q_{i+1}$. The sense of the tangent vector associated to this new vertex is chosen to be consistent with the sense of the tangent vectors associated to $Q_{i}$ and to $Q_{i+1}$. If this new vertex of $\mathcal{C}$ happens to be very close to $\mathcal{B}$, we may correct its slope as explained in the previous section. Further, the corresponding curvature value is 0 . In this way we reduce a non convex edge of $\mathcal{C}$ to the union of two consecutive convex edges.

\section{$3.3 \quad \varepsilon$-Error Controllability}

Definition 2. Given a magnitude $\varepsilon>0$, we call a section $\mathcal{A}_{i}$ of the $A$-spline $\mathcal{A}$ $\varepsilon$-controllable, if the points of $\mathcal{A}_{i}$ are at most at distance $\varepsilon$ to the corresponding edge $E_{i}:=\overline{Q_{i} Q_{i+1}}$ of $\mathcal{C}$.

In the present work, we will show sufficient conditions to ensure that the sections of the proposed A-spline scheme are $\varepsilon$-controllable. Note that if we use barycentric coordinates $(u, v, w)$ with respect to a triangle, such that the edge $E_{i}$ corresponds to the line $v=0$, then $\mathcal{A}_{i}$ is $\varepsilon$-controllable iff $|v| \leq \varepsilon_{i}$, for some $0 \leq \varepsilon_{i}$ depending on $\varepsilon$ and on the geometry of the triangle.

\section{Polygon Approximation by Cubic A-Spline Curves}

Given an ordered set of $n$ points in the plane, its associated polygon $\mathcal{C}$ and prescribed tangent vectors and curvature values at these points, we want to construct a cubic $G^{2}$-continuous A-spline curve $\mathcal{A}$, interpolating these points, as well as the direction and sense of their prescribed tangent vectors and curvature values. We may assume that all edges are convex (see subsection 3.2). 

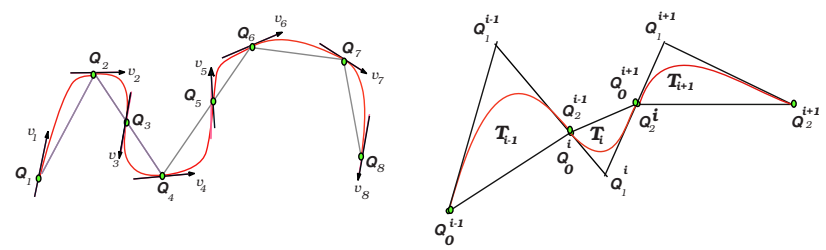

Fig. 2. Left: Interpolating points $Q_{i}$ with their prescribed tangent vectors $\overrightarrow{v_{i}}$. Right: Triangle chain.

\subsection{Triangle Chain}

Let us define a new sequence of points $Q_{j}^{i}$. First, set $Q_{0}^{i}:=Q_{i}$ and $Q_{2}^{i}:=Q_{i+1}$. Each pair of consecutive points $Q_{0}^{i}, Q_{2}^{i} \in \mathcal{C}$ with their tangent directions defines a triangle $T_{i}$ :

- If the tangent lines at $Q_{i}$ and at $Q_{i+1}$ are not parallel, then $T_{i}$ is the triangle with vertices. $Q_{0}^{i}, Q_{1}^{i}, Q_{2}^{i}$, where $Q_{1}^{i}$ is the point of intersection of the tangent lines at $Q_{0}^{i}:=Q_{i}$ and at $Q_{2}^{i}:=Q_{i+1}$.

- If $E_{i}:=\overline{Q_{i} Q_{i+1}}$ and the tangent lines at $Q_{i}$ and at $Q_{i+1}$ are all parallel, then $T_{i}$ is the degenerate triangle equal to $E_{i}$ and $Q_{1}^{i}$ is set equal to the midpoint of $E_{i}$.

- If the tangent lines at $Q_{i}$ and at $Q_{i+1}$ are parallel, but they are not parallel to the edge $E_{i}$, the midpoint $Q_{i}^{m}$ of the vertices $Q_{i}$ and $Q_{i+1}$ is inserted in $\mathcal{C}$ and as slope of the tangent line of $\mathcal{A}$ at this new vertex, it is assigned the slope of the line perpendicular to the bisector of the angle defined by $E_{i}$ and the tangent vector associated to $Q_{i}$. The sense of the tangent vector associated to $Q_{i}^{m}$ is chosen to be consistent with the sense of the tangent vectors associated to $Q_{i}$ and to $Q_{i+1}$. Then, the sequence of points $Q_{j}^{k}$ for $k=i, j=2$ and for $k>i$ is updated and a triangle to each of the two new pairs of consecutive vertices of $\mathcal{C}$ (corresponding to $Q_{i}$ and $Q_{i}^{m}$, respectively to $Q_{i}^{m}$ and $\left.Q_{i+1}\right)$ a triangle is assigned as explained above.

\section{$4.2 \quad G^{1}$-Continuity}

In order to obtain a continuous curve $\mathcal{A}$ ( $G^{0}$-continuity), we must require that $Q_{2}^{i}=Q_{0}^{i+1}$ for $i=1, \ldots, n-1$. If the curve is closed, it is necessary that $Q_{2}^{n}=Q_{0}^{1}$.

$\mathcal{A}_{i}$ may be written in barycentric coordinates $(u, v, w), w=1-u-v$ with respect to the vertices of $T_{i}$ as,

$$
\mathcal{A}_{i}: f_{i}(u, v)=\sum_{j=0}^{3} \sum_{k=0}^{3-j} a_{k, j}^{i} u^{k} v^{j} w^{3-k-j}=0
$$

It is well known that $\mathcal{A}_{i}$ interpolates $Q_{0}^{i}$ and $Q_{2}^{i}$ iff the coefficients $a_{0,0}^{i}$ and $a_{3,0}^{i}$ in (11) vanish. Furthermore, the tangent lines to $\mathcal{A}_{i}$ at $Q_{0}^{i}$ and $Q_{2}^{i}$ are the corresponding sides of the triangle $T_{i}$ iff $a_{0,1}^{i}$ and $a_{2,1}^{i}$ vanish. 
Assuming that the previous restrictions on the points $Q_{j}^{i}$ as well as on the coefficients of (11) are satisfied, then $\mathcal{A}$ is $G^{1}$-continuous.

\subsection{Explicit Expressions for $\mathcal{A}_{i}$}

The A-spline curve $\mathcal{A}$ discussed in this paper consists of a chain of curve sections $\mathcal{A}_{i}$. Each section is defined as the zero contour of a bivariate BB-polynomial of degree 3 . We show that these curve sections are convex, connected and nonsingular in the interior of the regions of interest. For $-\frac{1}{2}<\delta_{i}$, the explicit expression for $\mathcal{A}_{i}$ is:

$$
\mathcal{A}_{i}:-v^{3}+\frac{u w(u+w)-\left(\kappa_{2}^{i} w+\kappa_{0}^{i} u\right) v^{2}}{2} \delta_{i}{ }^{3}+\frac{\left(\kappa_{0}^{i}+\kappa_{2}^{i}\right) u v w}{2} \delta_{i}{ }^{4}=0
$$

If $\delta_{i}=0$, section $\mathcal{A}_{i}$ represents the line segment joining $Q_{0}^{i}$ to $Q_{2}^{i}$. That is the case when triangle $T_{i}$ is equal to the edge $E_{i}$.

Since each section $\mathcal{A}_{i}$ is traced out from the initial point $Q_{0}^{i}$ to the final point $Q_{2}^{i}$ then, if $\delta_{i} \neq 0$, according to the sense of vector $\overrightarrow{v_{i}}$ associated to $Q_{0}^{i}$ we must consider two cases:

- Inner case: $\overrightarrow{v_{i}}$ points out to the halfplane containing $Q_{1}^{i}$.

- Outer case: $\overrightarrow{v_{i}}$ does not point out to the halfplane containing $Q_{1}^{i}$.

In the inner case, section $\mathcal{A}_{i}$ is the zero contour of the cubic curve with equation given in $(2)$ and $0<\delta_{i}$. In this case, the interior of the triangle $\{(u, v, w): 0<$ $u, v, w<1, u+v+w=1\}$ is called the region of interest and it is denoted by $\Omega_{i}$. In the outer case, section $\mathcal{A}_{i}$ is the zero contour of the cubic curve with equation given in $(2)$ and $-\frac{1}{2}<\delta_{i}<0$. In this case, the region of interest is equal to $\{(u, v, w):-1<v<0, \quad 0<u, w<2, \quad 0<u<w\}$ and it is also denoted by $\Omega_{i}$.

Theorem 1. For $\delta_{i} \neq 0,-\frac{1}{2}<\delta_{i}$, the plane cubic curves $\mathcal{A}_{i}$ satisfy the following properties:

1. They interpolate the points $Q_{j}^{i}, j=0,2$. The tangent lines at $Q_{j}^{i}$ are the corresponding sides of $T_{i}$.

2. At $Q_{j}^{i}, j=0,2$ they have curvature value $k_{j}^{i}=\frac{\kappa_{j}^{i} \Delta_{i}}{\left(g_{j}^{i}\right)^{3}}$. Here $g_{j}^{i}=\left\|Q_{j}^{i}-Q_{1}^{i}\right\|$ and $\Delta_{i}$ denotes the area of $T_{i}$.

3. In the interior of the corresponding region of interest $\Omega_{i}, \mathcal{A}_{i}$ are non singular, connected and convex.

4. If $\left|\varepsilon_{i}\right| \geq 1$ then, curves $\mathcal{A}_{i}$ are $\varepsilon$-controllable. Otherwise, for $\left|\delta_{i}\right| \leq \frac{\varepsilon_{i}}{1-\varepsilon_{i}}$, $\mathcal{A}_{i}$ are $\varepsilon$-controllable.

Proof. We will only present the main arguments:

1. The coefficients of section $\mathcal{A}_{i}$ satisfy all the vanishing conditions given in subsection 4.3 . 
2. If we apply to $\mathcal{A}_{i}$ the known formulas for the curvature of implicitly defined curves (see for instance [5] or [1]), we obtain at its endpoints $Q_{j}^{i}, j=0,2$, the above mentioned formulas linking the curvature values $k_{j}^{i}, j=0,2$ at these endpoints and the geometry of $T_{i}$ with the parameters $\kappa_{j}^{i}, j=0,2$ of $\mathcal{A}_{i}$. It provides a local control of the curvature at the knots of the A-spline.

3. For the outer case, the known results for the inner case (see [5] or [1], for instance) do not apply. Let's consider the subset $\widetilde{\mathcal{L}}$ of the pencil $\mathcal{L}$ of lines passing through $Q_{1}^{i}$ defined as $\widetilde{\mathcal{L}}:=\left\{l \in \mathcal{L}: l \bigcap \Omega_{i} \neq \emptyset\right\}$. The value of the $v$-coordinate of the intersection of each line $l \in \widetilde{\mathcal{L}}$ with any of the curves $\mathcal{A}_{i}$ satisfies a cubic equation. After reparametrizing and writing this cubic equation in BB-form, it is possible to apply the variation diminishing principle. This permits us to show that in the interior of the region of interest $\Omega_{i}$, $l$ and each curve $\mathcal{A}_{i}$ have only one intersection point, counting multiplicity. Hence, these curves are connected and non singular in the interior of $\Omega_{i}$.

Assuming the existence of an inflection point in $\Omega_{i}$, since the curves $\mathcal{A}_{i}$ have just been shown to be connected and since they are convex in a neighborhood of $Q_{0}^{i}$ as well as of $Q_{2}^{i}$, then there are at least two inflection points in $\Omega_{i}$. Thus, considering the line passing through two consecutive inflexion points in $\Omega_{i}$, it is straightforward to show that this line cuts $\mathcal{A}_{i}$ at least in 4 points. But $\mathcal{A}_{i}$ are cubic curves, hence we get a contradiction to Bezout's Theorem.

4. For fixed $\delta_{i} \neq 0$, let's consider the limit cases $\mathcal{A}_{i}^{j}$ ( for $\kappa_{j}^{i} \rightarrow \infty, j=0,2$ ) and $\mathcal{A}_{i}^{00}$ ( for $\kappa_{0}^{i}=\kappa_{2}^{i}=0$ ). It is not difficult to realize that the equation of $\mathcal{A}_{i}$ is a convex linear combination of the equations of these 3 limit cases. The $v$-coordinate of the relative extremes of $v=v(u)$ on the graph of the limit curves are attained at the $v$-value $\frac{\delta_{i}}{1+\delta_{i}}$, for $\mathcal{A}_{i}^{j}, j=0,2$ and at the $v$-value $\frac{\delta_{i}}{2+\delta_{i}}$, for $\mathcal{A}_{i}^{00}$. Hence, the $v$-coordinate of the relative extremes of $v=v(u)$ on the graph of $\mathcal{A}_{i}$ is bounded above by $\frac{\delta_{i}}{1+\delta_{i}}$ for $\delta_{i}>0$ and it is bounded below by $\frac{\delta_{i}}{1+\delta_{i}}$ for $\delta_{i}<0$. Therefore, for $\left|\varepsilon_{i}\right|<1$, imposing $\left|\delta_{i}\right| \leq \frac{\varepsilon_{i}}{1-\varepsilon_{i}}$, we obtain the proof of the $\varepsilon$-controllability of $\mathcal{A}_{i}$.

\section{$4.4 G^{2}$-Continuity}

We already have shown that $\mathcal{A}$ is $G^{1}$-continuous. The above proposed cubic sections $\mathcal{A}_{i}$ have, by construction, free parameters $\kappa_{j}^{i}, j=0,2$ that permit us to set the curvature value of $\mathcal{A}_{i}$ at $Q_{i}$ equal to the curvature values estimated at each vertex $Q_{i}$ of $\mathcal{C}$ in the subsection 3.1. Hence, $\mathcal{A}$ is $G^{2}$-continuous.

\subsection{Avoiding the Boundary $\mathcal{B}$}

Since the sections $\mathcal{A}_{i}$ of the A-spline $\mathcal{A}$ interpolate the data $Q_{i}$ as well as the prescribed slope and curvature values at these points, it only remains to decide which values for the free parameter $\delta_{i}$ provide an arc that does not cross $\mathcal{B}$.

If the intersection of the region of interest $\Omega_{i}$ with $\mathcal{B}$ is void, we may choose freely the value of $\delta_{i}$, for instance, we may set $\delta_{i}=-\frac{1}{4}$ in the outer case and 

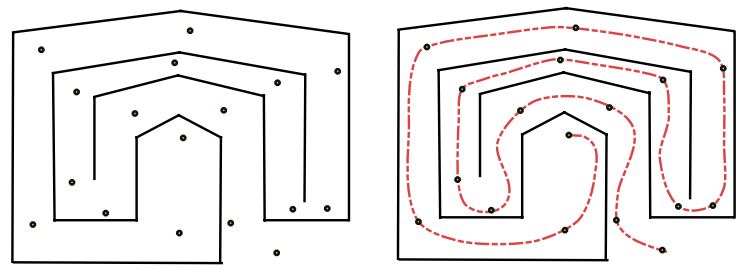

Fig. 3. Interpolation of data avoiding the boundary

$\delta_{i}=1$ in the inner case. Otherwise, since the Hausdorff distance $d_{i}$ between the line segment $E_{i}:=\overline{Q_{i} Q_{i+1}}$ and the set $\mathcal{B} \cap \Omega_{i}$ is attained at a subset of the vertices of $\mathcal{B}$, it is easy to compute $d_{i}$. This value of distance $d_{i}$ in real coordinates corresponds to a value $\varepsilon_{i}$ in the barycentric coordinates associated to triangle $T_{i}$. If $\left|\varepsilon_{i}\right| \geq 1$, for any value of $\delta_{i}>-\frac{1}{2}$ section $\mathcal{A}_{i}$ does not cross $\mathcal{B}$, else impose $\left|\delta_{i}\right|<\frac{\varepsilon_{i}}{1-\varepsilon_{i}}$.

\section{Conclusions}

The proposed A-spline scheme is $G^{2}$-continuous with degree 3 and has the minimal number of inflection points consistent with the data.

In comparison to [2, we do not impose restrictions for the interpolation of tangent vectors. On the other hand, we interpolate not only the directions of the tangent vectors but also their sense, which is a completely new feature in the A-spline context.

In comparison to 4, the corrections of the slope values presented in the current work generalize the adaptation to the constrained case suggested by these authors. In our approach, imposing $G^{2}$-continuity and avoiding the boundary $\mathcal{B}$ to each segment is achieved locally and at the same time (there is no need of changing the free parameters of preceding and succeeding segments in order to restore $G^{2}$-continuity). Moreover, it is simpler to prevent the spline curve from crossing $\mathcal{B}$ using the $\varepsilon$-controllability of our cubic A-spline scheme.

\section{Acknowledgements}

The authors wish to thank to R. Patterson and also to the anonymous referees for their very useful suggestions and comments, which have helped us to improve the presentation of this work. The results exposed in this contribution were obtained with the support of the grant PNCB/04-04.

\section{References}

1. Bajaj, C., Xu, G.: A-Splines: Local Interpolation and Approximation using $G^{k}$ Continuous Piecewise Real Algebraic Curves. CAGD 16, 557-578 (1999)

2. Bajaj, C., Xu, G.: Regular algebraic curve sections (III) - Applications in interactive design and data fitting. CAGD 18, 149-173 (2001) 
3. Goodman, T.N.T.: Shape preserving interpolation by parametric rational cubic splines. In: International Series of Numerical Mathematics, vol. 86, pp. 149-158. Birkhäuser Verlag, Basel (1988)

4. Meek, D.S., Ong, B.H., Walton, D.J.: Constrained interpolation with rational cubics. CAGD 20, 253-275 (2003)

5. Paluszny, M., Patterson, R.: $G^{2}$-continuous cubic algebraic splines and their efficient display. In: Laurent, P.J., Le Méhauté, A., Schumacker, L.L. (eds.) Curves and Surfaces II, pp. 353-359 (1994)

6. Paluszny, M., Patterson, R.: Geometric control of $G^{2}$-cubic A-splines. CAGD 15, 261-287 (1998) 